DOMINGOS, H.G.T. et al. Influência do sombreamento e aspersão de água sobre a produção de leite e respostas fisiológicas de vacas leiteiras. PUBVET, Londrina, V. 6, N. 9, Ed. 196, Art. $1318,2012$.

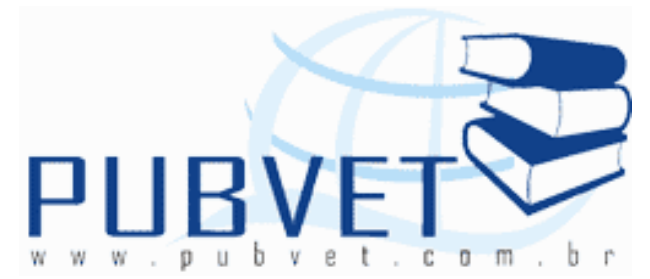

PUBVET, Publicações em Medicina Veterinária e Zootecnia.

\title{
Influência do sombreamento e aspersão de água sobre a produção de leite e respostas fisiológicas de vacas leiteiras
}

\author{
Hérica Girlane Tertulino Domingos ${ }^{1}$, João Batista Freire de Souza Junior ${ }^{1}$, Maiko \\ Roberto Tavares Dantas ${ }^{1}$, Geovan Figueirêdo Sá Filho ${ }^{1}$, Janio Lopes Torquato ${ }^{1}$, \\ Leonardo Lelis de Macedo Costa ${ }^{1}$
}

1 Universidade Federal Rural do Semi-Árido, Departamento de Ciências Animais, Laboratório de Biometeorologia, Biofísica Ambiental e Bem-Estar Animal (LABBEA), BR 110, Km 47, Costa e Silva, CEP: 59625-900, MossoróRN.

\section{Resumo}

Inúmeros trabalhos atestam os efeitos negativos das elevadas temperaturas sobre a produção de leite. Por isso diversas modificações ambientais podem ser introduzidas, visando diminuir a temperatura sobre os animais e, conseqüentemente, atenuar o estresse por calor, auxiliando, assim, no conforto de vacas leiteiras confinadas. Assim, o objetivo desta revisão foi abordar os efeitos do sombreamento e da aspersão de água sobre a produção de leite e respostas fisiológicas em vacas leiteiras. Vários métodos têm sido empregados, tais como: sombreamento natural ou artificial, ventilação, resfriamento do ar a partir da aplicação de gotículas de água, pulverização de água sobre os animais acompanhada de ventilação e condicionamento do ar. Em sistemas de produção intensiva, somado ao sombreamento, a utilização de 
DOMINGOS, H.G.T. et al. Influência do sombreamento e aspersão de água sobre a produção de leite e respostas fisiológicas de vacas leiteiras. PUBVET, Londrina, V. 6, N. 9, Ed. 196, Art. $1318,2012$.

sistemas de resfriamento evaporativo proporcional aos animais um ambiente com temperaturas mais amenas, evitando situações de estresse térmico e aumentando a produção leiteira. Já em pastagens, o fornecimento de sombra, seja natural ou artificial, torna-se necessário para vacas leiteiras com o objetivo de diminuir o excesso de calor recebido através da radiação solar direta, principalmente nas horas mais quentes do dia e em regiões próximas a linha do Equador.

Palavras-chave: ambiente tropical, produção de leite, resfriamento evaporativo, sombreamento

\title{
Influence of shading and sprinkling water on milk production and physiological responses of dairy cows
}

\begin{abstract}
Countless studies attest to the negative effects of high temperatures on milk production. So, many environmental modifications can be introduced in order to decrease the temperature of the animals and, consequently, reduce heat stress, helping so, in the comfort of dairy cows confined. The aim of this review was to approach the effects of shading and sprinkling water on milk production and physiological responses in dairy cows. Several methods have been employed, such as natural or artificial shading, ventilation, cooling air from the application of water droplets, water spray on the animal accompanied by ventilation and air conditioning. In intensive production systems, coupled with the shading, the use of evaporative cooling systems in proportion to the animals an environment with warmer temperatures, avoiding situations of heat stress and increasing milk production. In pastures, the providing shade, either natural or artificial, it is necessary for dairy cows in order to reduce the excess heat received by direct solar radiation, especially during the hottest hours of the day and in regions near the line of Ecuador.
\end{abstract}

Keywords: tropical environment, milk production, evaporative cooling, shading 
DOMINGOS, H.G.T. et al. Influência do sombreamento e aspersão de água sobre a produção de leite e respostas fisiológicas de vacas leiteiras. PUBVET, Londrina, V. 6, N. 9, Ed. 196, Art. $1318,2012$.

\section{INTRODUÇÃO}

A pecuária é uma atividade altamente dependente dos fatores climáticos, cujas alterações podem afetar a produtividade e o manejo dos animais. As condições de adaptação dos sistemas de produção de leite à mudança do clima podem ser variáveis, colocando-os em posições mais, ou menos vulneráveis em função dos diferentes cenários climáticos. Regiões tropicais e subtropicais como é o caso do Brasil, podem tornar-se extremamente desconfortáveis para as vacas leiteiras de raças especializadas, principalmente para aquelas em lactação.

Em ambientes tropicais, a expressão do potencial de produção dos animais é restringida pelo efeito de fatores ambientais tais como altas temperaturas e intensa radiação solar. Estes reduzem no animal, a capacidade de eliminar calor corporal, resultando em uma condição conhecida como estresse calórico que traz como resposta, redução de consumo de alimentos e da taxa metabólica, aumento da temperatura retal e da freqüência respiratória, aumento do consumo de água, alterações das concentrações hormonais, aumento da sudorese e alterações das necessidades de mantença. Finalmente, estes mecanismos resultam em redução da produção de leite, e conseqüentemente perdas econômicas significativas para o produtor.

Porém, dentro da zona de conforto térmico ou de termoneutralidade, que é limitada pela máxima e mínima temperatura ótima para a produção, o animal mantém uma variação normal de temperatura corporal e de freqüência respiratória, o apetite é normal e a produção é ótima (BACCARI JR el al., 1997).

Segundo Silva (1999), não há uma tecnologia padrão que possa ser utilizada em todos os países, nem em todo o território nacional, pois para sua utilização deve-se considerar a raça dos animais, a fase de desenvolvimento, o nível tecnológico, o nível de produção, as características climáticas e de relevo das regiões, os sistemas e instalações já existentes, entre outros fatores. As alternativas para manter ou melhorar o desempenho e o bem-estar dos 
DOMINGOS, H.G.T. et al. Influência do sombreamento e aspersão de água sobre a produção de leite e respostas fisiológicas de vacas leiteiras. PUBVET, Londrina, V. 6, N. 9, Ed. 196, Art. $1318,2012$.

animais em climas quentes envolvem, necessariamente, os processos metabólicos de utilização de energia e sua transferência através dos processos de trocas de calor entre o animal e o ambiente (BAÊTA, 1998). Assim diversas modificações ambientais podem ser introduzidas, tais como, sombreamento natural ou artificial, ventilação, resfriamento do ar a partir da aplicação de gotículas de água, aspersão de água sobre os animais acompanhada ou não, de ventilação e condicionamento do ar (BUCKLIN et al., 1990; FRAZZI et al., 1998).

Esses resultados mostram que oferecer um ambiente climatizado pode melhorar a qualidade do microclima no interior das instalações, atenuando o estresse por calor, auxiliando, assim, no conforto de vacas leiteiras e conseqüentemente evitando perdas produtivas. Assim, o objetivo desta revisão foi abordar os efeitos do sombreamento e da aspersão de água sobre a produção de leite e respostas fisiológicas em vacas leiteiras.

\section{Respostas fisiológicas}

Os ruminantes são animais classificados como homeotermos, ou seja, apresentam funções fisiológicas que se destinam a manter a temperatura corporal constante, controlada pelo centro termorregulador no sistema nervoso central, acionado principalmente, por células termo-receptoras localizado na pele. Informações externas do ambiente são captadas por estas células e levadas ao hipotálamo, que aciona mecanismos fisiológicos para dissipação ou conservação do calor (CURTIS, 1983).

Dentro de certa faixa de temperatura ambiente, determinada zona de conforto ou de termoneutralidade, isso ocorre com mínima mobilização dos mecanismos termorreguladores. Nesta situação o animal não sofre estresse por calor ou frio e ocorre mínimo desgaste além de melhores condições de saúde e de produtividade (NÄÄS, 1989; TITTO, 1998).

Não há concordância absoluta entre os autores acerca dos limites de zona de termoneutralidade. Para Nääs (1989), zona de conforto seria aquela 
DOMINGOS, H.G.T. et al. Influência do sombreamento e aspersão de água sobre a produção de leite e respostas fisiológicas de vacas leiteiras. PUBVET, Londrina, V. 6, N. 9, Ed. 196, Art. $1318,2012$.

limitada pela máxima e mínima temperatura ótima para a produção. A mesma autora enfatizou que essas zonas de conforto deveriam ser encaradas como uma indicação e analisadas acerca de sua aplicabilidade às condições especificas e projetadas à realidade de cada ambiente, merecendo uma série de estudos e pesquisas.

Esmay (1982) estabeleceu que a quantidade de calor trocado entre o animal e sua circunvizinhança depende das condições termodinâmicas do ambiente. Se a temperatura é maior ou menor que a faixa estabelecida como ótima de conforto, o sistema termorregulador é ativado para manter o equilíbrio térmico entre o animal e o meio.

De acordo com (COPPOCK e WEST, 1986) apesar de ser o meio natural de controle da temperatura do organismo, a termorregulação representa um esforço extra e, conseqüentemente uma alteração na produtividade. A manutenção da homeotermia é prioridade para os animais, imperando sobre as funções produtivas.

Respostas fisiológicas de vacas leiteiras, sob condição de estresse térmico por calor, normalmente são: aumento da temperatura retal; redução na ingestão de alimentos; aumento da freqüência respiratória; aumento da sudorese; e redução da produção; além de modificações hormonais e baixa na fertilidade. De forma geral, o gado leiteiro sob condições térmicas estressantes aumenta a dissipação de calor na forma evaporativa (transpiração e ofegação) e reduz a quantidade de calor resultante de processos metabólicos (produção) (SILVA, 2000).

O aumento da freqüência respiratória é o primeiro sinal visível de animais submetidos ao estresse térmico, embora este seja o terceiro na seqüência dos mecanismos de termorregulação. O primeiro mecanismo é a vasodilatação e o segundo, a sudação. O aumento ou a diminuição da freqüência respiratória está na dependência da intensidade e da duração do estresse a que estão submetidos os animais. Esse mecanismo fisiológico promove a perda de calor por meio evaporativo. 
DOMINGOS, H.G.T. et al. Influência do sombreamento e aspersão de água sobre a produção de leite e respostas fisiológicas de vacas leiteiras. PUBVET, Londrina, V. 6, N. 9, Ed. 196, Art. 1318, 2012.

Alterações na freqüência respiratória são usadas por diversas espécies animais para trocar calor com o ambiente pela via respiratória; o seu aumento tem sido descrito por vários autores (GAYÃO, 1992) como sendo a primeira resposta corporal à elevação da temperatura do ar.

Os valores normais de freqüência para bovinos leiteiros adultos da raça holandesa situam-se entre 10 e 40 mov.mim ${ }^{-1}$ (RODRIGUEZ, 1948 ; KELLY, 1967). Segundo Hahn et al. (1997), entretanto, a freqüência de $60 \mathrm{mov} \cdot \mathrm{min}^{-1}$ indica animais com ausência de estresse térmico ou que este é mínimo; mas, quando ultrapassam 120 mov. $\mathrm{min}^{-1}$, refletem cargas excessiva de calor.

Estudos confrontando vacas em lactação com exposição ao sol contra animais totalmente sombreados, relataram redução na freqüência respiratória e aumento na produção de leite (COLLIER et al, 1981; ROMAN-PONCE et al, 1977) já Baccari Jr. et al. (1982) observaram efeitos do sombreamento apenas sobre as variáveis fisiológicas (temperatura retal e freqüência respiratória), enquanto a produção de leite não variou. Segundo o autor, este fato pode ter ocorrido em razão do baixo nível produtivo dos animais avaliados.

A medida da temperatura retal é usada freqüentemente como índice de adaptabilidade fisiológica aos ambientes quentes, pois seu aumento mostra que os mecanismos de liberação de calor tornaram-se insuficientes (MOTA, 1997). De acordo com McDowell et al. (1976) a temperatura retal normal aceita para todas as raças bovinas é de $38,33^{\circ} \mathrm{C}$, com alguma variação de acordo com a raça, idade, estagio de lactação, nível nutricional e estagio reprodutivo. Em estudos de tolerância ao calor em novilhas da raça Holandesa, analisou-se a temperatura retal à sombra e ao sol, e foi concluído que a elevação da temperatura do ar induziu, em todas as raças, um aumento da temperatura retal.

A temperatura da superfície corporal dos animais influencia diretamente a troca de calor sensível, sobre altas temperaturas, esses mecanismos podem se transformar numa via de ganho de calor (MAIA et al., 2005). Esta, depende diretamente das condições do meio, ou seja, alterações na quantidade de radiação incidente, principalmente se o animal for exposto a céu aberto, 
DOMINGOS, H.G.T. et al. Influência do sombreamento e aspersão de água sobre a produção de leite e respostas fisiológicas de vacas leiteiras. PUBVET, Londrina, V. 6, N. 9, Ed. 196, Art. $1318,2012$.

alteram rapidamente a temperatura dessa superfície. De acordo com Silva et al, (2008) a temperatura da superfície do pelame depende diretamente das condições do meio, ou seja, alterações na quantidade de radiação incidente, principalmente se o animal for exposto a céu aberto, alteram rapidamente a temperatura dessa superfície.

\section{Sombreamento}

O fornecimento de sombra, seja natural ou artificial, torna-se necessário para vacas leiteiras com o objetivo de diminuir o excesso de calor recebido através da radiação solar direta, principalmente nas horas mais quentes do dia e em regiões próximas a linha do Equador, onde os níveis de radiação podem ultrapassar os $1000 \mathrm{~W} \mathrm{~m}^{2}$ entre $10 \mathrm{~h}$ e $15 \mathrm{~h}$ na região semiárida do Brasil, como foi descrito por Silva et al. (2010).

A temperatura ótima para a lactação depende da espécie, da raça e de sua capacidade de tolerância ao calor ou frio. Dentro de uma faixa determinada de temperatura as produções permanecem relativamente inalteradas (máximas e mínimas). A literatura afirma que a temperatura crítica para produção de leite, varia com o tamanho do animal e o nível de produção láctea. Os custos fisiológicos mínimos e a produtividade máxima costumam ocorrer quando os animais estão na zona de termoneutralidade (BERMAN et al., 2008) que para vacas holandesas está entre $-5^{\circ} \mathrm{C}$ e $21^{\circ} \mathrm{C}$ (JOHNSON, 1987).

As vacas de raças leiteiras, quando em lactação, são particularmente sensíveis ao estresse térmico devido possivelmente a sua função produtiva especializada e a sua alta eficiência na utilização dos alimentos. Os animais absorvem calor do ambiente além daquele produzido no organismo (metabolismo energético), sendo que, durante o dia, quase todo o calor absorvido do ambiente pelos animais provém da radiação solar, direta ou indireta. 
DOMINGOS, H.G.T. et al. Influência do sombreamento e aspersão de água sobre a produção de leite e respostas fisiológicas de vacas leiteiras. PUBVET, Londrina, V. 6, N. 9, Ed. 196, Art. 1318, 2012.

De acordo com Silva (2000), a proteção proporcionada pela sombra é uma barreira contra a radiação solar direta, e não contra o calor propriamente dito, já que essa proteção não altera a temperatura do ar. Dados os elevados níveis de radiação solar nas zonas intertropicais, essa proteção é essencial e há inúmeras pesquisas que comprovam que a simples existência de sombra de arvores nos pastos pode alterar favorável e significativamente o desempenho dos animais, como comprovou Roman-ponce et al. (1977) que observaram uma melhoria de 10,7\% na produção de vacas holandesas, Guernseys e Pardosuíças com acesso à sombra. Collier et al. (1981) obtiveram resultados similares com vacas Jersey e Holandesas. Por outro lado, Baccari Jr et al. (1982) observaram 28 vacas leiteiras durante o verão em Botucatu (SP), não encontrando diferenças significativas quanto à produção de vacas mantidas à sombra e vacas sujeitas à insolação. Tratava-se de animais mestiços azebuados, que sem duvidas apresentam boa tolerância à radiação solar.

Damasceno et al. (1998) compararam dois sistemas de sombreamento em currais tipo "free-stall", com ou sem cobertura de lona plástica nas extremidades sudeste-noroeste da área coberta. Concluíram que vacas com acesso ao curral com cobertura de lona plástica apresentam freqüências respiratórias e temperaturas retais inferiores. Também a produção de leite das vacas, com acesso ao curral com lona, foi superior às demais com acesso ao curral sem essa cobertura.

Trabalhos realizados em regiões de clima quente tem demonstrado que vacas que dispõem de acesso à sombra no verão podem produzir até $21,5 \%$ a mais de leite que suas congêneres mantidas ao sol durante as horas mais quentes do dia. Desta forma conclui-se que, a sombra pode reduzir de 30 a $50 \%$ a carga de calor sobre os animais. Armstrong et al. (1993) mostram que a sombra para vacas leiteiras é considerada essencial para minimizar a perda na produção de leite e na eficiência reprodutiva.

Bond et al. (1967) confirmam que uma simples sombra interceptando os raios solares pode reduzir o calor radiante no animal em aproximadamente $30 \%$ ou mais. 
DOMINGOS, H.G.T. et al. Influência do sombreamento e aspersão de água sobre a produção de leite e respostas fisiológicas de vacas leiteiras. PUBVET, Londrina, V. 6, N. 9, Ed. 196, Art. $1318,2012$.

\section{Aspersão de Água}

Inúmeros trabalhos atestam os efeitos negativos das elevadas temperaturas sobre a produção de leite. Por isso diversas modificações ambientais podem ser introduzidas, visando diminuir a temperatura sobre os animais e, conseqüentemente, atenuar o estresse por calor, auxiliando, assim, no conforto de vacas leiteiras confinadas. Vários métodos têm sido empregados, tais como: sombreamento natural ou artificial, ventilação, resfriamento do ar a partir da aplicação de gotículas de água, pulverização de água sobre os animais acompanhada de ventilação e condicionamento do ar (BUCKLIN et al., 1990; ARMSTRONG et al., 1993; ARMSTRONG, 1994).

O sistema de resfriamento adiabático evaporativo (SRAE), é uma outra medida de incremento do desempenho dos animais. Este, expandiu-se rapidamente em climas quentes, devido à sua simplicidade técnica, praticidade e relação custo/beneficio favorável, resultado em aceitação por muitos, podendo ser obtido por vários processos, entre os quais se destacam: a nebulização, a microaspersão e a aspersão sobre os animais ou telhados.

A aspersão sobre os animais não tem por finalidade resfriar o ar, pois emprega gotas com diâmetro maior, de modo a promover o umedecimento dos pêlos e epiderme das vacas. O animal se resfria com a evaporação da água, por meio da pele e pelos, permitindo a troca de calor mais eficiente, quando comparada a apenas com a sudação (BUCKLIN e BRAY, 1998).

A aspersão direta de água sobre as vacas conduz o calor da superfície corporal para o ambiente, e leva os animais a evaporarem mais umidade da pele permitindo que as vacas utilizem o resultante calor latente de evaporação para o resfriamento do corpo. Isto faz com que diminuam as compensações induzidas pelo calor como o decréscimo na ingestão de alimentos, alterações hormonais e outros fatores que resultam numa produção de leite diminuída, de modo que a energia utilizada para os processos de resfriamento do corpo possa ser poupada para as funções produtivas. 
DOMINGOS, H.G.T. et al. Influência do sombreamento e aspersão de água sobre a produção de leite e respostas fisiológicas de vacas leiteiras. PUBVET, Londrina, V. 6, N. 9, Ed. 196, Art. 1318, 2012.

Na Austrália subtropical, vacas holandesas de alta produção, confinadas, com livre acesso à sombra e aspergidas com água sempre que a temperatura do ar excedeu $26^{\circ} \mathrm{C}$, produziram $4,8 \mathrm{~kg} \mathrm{~d}-1$ a mais de leite que seus pares sem aspersão de água (HALL et al., 1997). Igono et al. (1985) utilizaram aspersão de água em vacas holandesas durante o verão, no Estado de Colúmbia (EUA) e encontraram acréscimos de $0,7 \mathrm{~kg}$ de leite quando as vacas foram comparadas com as sem aspersão. Em trabalhos realizados com vacas em lactação nos Emirados Árabes, segundo Igono et al. (1985), as perdas de produção de leite, devido ao verão, foram reduzidas em $2,8 \%$, com o uso de aspersão de água, sendo que, no total de 305 dias de lactação, representaram 0,6\%.

De acordo com Bucklin e Bray (1998), o principal benefício do uso da aspersão sobre animais, é que, além de aumentar a capacidade de perda de calor através de evaporação, também está baseado no aumento de consumo de alimento e conseqüente aumento de produção. Igono (1985) trabalhando com sistemas de aspersão em vacas holandesas no Missouri encontrou um aumento de $700 \mathrm{~g}$ a mais de leite por dia. Para o mesmo tratamento, Hall et al. (1997) observou um acréscimo de $4,8 \mathrm{~kg} \mathrm{~d}^{-1}$ a mais de leite quando as vacas foram comparadas com as sem aspersão. Em estudos realizados por Perissinotto et al. (2003), observou-se que as perdas de produção de leite, com o uso de aspersão de água foram reduzidas em $2,8 \%$, sendo que, no total de 305 dias de lactação, representaram 0,6\%.

Uma outra medida de incremento do desempenho dos animais, é a aspersão de água, que segundo Nääs (1989), até um limite aproximado de umidade relativa do ar de $70 \%$, é a melhor maneira de se resfriar um ambiente destinado a animais em lactação, uma vez que a mesma possui alta capacidade calorífica e elevado calor latente de vaporização. Nesses sistemas, a água deve penetrar e umedecer completamente a pele e o pêlo dos animais, de forma que as vacas sejam resfriadas e troquem calor por condução e por evaporação da água, a partir dos pêlos e da pele. 
DOMINGOS, H.G.T. et al. Influência do sombreamento e aspersão de água sobre a produção de leite e respostas fisiológicas de vacas leiteiras. PUBVET, Londrina, V. 6, N. 9, Ed. 196, Art. $1318,2012$.

\section{Considerações finais}

Em pastagens, o fornecimento de sombra, seja natural ou artificial, torna-se necessário para vacas leiteiras com o objetivo de diminuir o excesso de calor recebido através da radiação solar direta, principalmente nas horas mais quentes do dia e em regiões próximas a linha do Equador. Em sistemas de produção intensiva, somado ao sombreamento, a utilização de sistemas de resfriamento evaporativo proporcional aos animais um ambiente com temperaturas mais amenas, evitando situações de estresse térmico e aumentando a produção leiteira.

\section{REFERÊNCIAS}

ARCARRO JR., ARCARRO, J. R. P.; POZZI, C. R.; MATARAZZO, S. V.; FAGUNDES, H. Produção e composição do leite de vacas holandesas em sala de espera climatizada. In: CONGRESSO BRASILEIRO DE BIOMETEOROLOGIA, 3., Maringá, 2001. Anais... Maringá:, 2001.

ARMSTRONG, D.V., W.T. WELCHERT AND F. WIERSMA. Environmental modification for dairy cattle housing in arid climates. In: Proc. 4th Int. Livestock Environmental Symp., Warwick, Coventry, England. Am. Soc. Agric. Eng., St. Joseph, MI, USA, 1993.

ARMSTRONG, D.V. Heat Stress Interaction with Shade and Cooling. J. Dairy Sci. 77:20442050, 1994.

BACCARI JR., F.; ASSIS, P.S.; POLASTRE, R. et al. Shade management in tropical environment for milk production in crossbred cows. Proceedings Western Section American Society of Agricultural Engineering, v.33, p.209-210, 1982.

BACCARI JR., BRASIL, L.H.A., TEODORO, S.M. et al. Thermoregulatory responses of Alpine goats during thermal stress. In: LIVESTOCK ENVIRONMENT, 1997, Minneapolis. Proceedings... Minneapolis: ASAE, 1997. p.789-794.

BERMAN, A. Increasing heat stress relief produced by coupled coat wetting and forced ventilation. Journal Dairy Science, v.91, p. 4571, 2008.

BOND, T. E., C. F. KELLY, S. R. MORRISON, AND N. PEREIRA. 1967. Solar, atmospheric, and terrestrial radiation received by shaded and unshaded animals. Trans. Am. Soc. Agric. Eng. 10(5) :622-62, 1967.

BUCKLIN, R.A.; BRAY, D.R. The american experience in dairy management in warm and hot climates. In: SIMPÓSIO BRASILEIRO DE AMBIÊNCIA NA PRODUÇÃ̃O DE LEITE, 1., 1998, Piracicaba. Anais... Piracicaba:FEALQ, 1998, p.156-174. 
COLLIER, R.J.; ELEY, R.M.; SHARMA, A.K. et al. Shade management in subtropical environment for milk yield and composition in Holstein an Jersey cows. Journal of Dairy Science, v.64, p.844-849, 1981.

COPPOCK, C. E. AND J. W. WEST. 1986. Nutritional adjustments to reduce heat stress in lactating dairy cows. In: Proc. Georgia Nutr. Conf. Feed Industry. Atlanta. GA.Univ. Georgia, Athens. GA. pp. 19-26.

CURTIS, S.E. Environmental management in animal agriculture. Ames : The Iowa State University, 1983. 409p.

DAMASCENO, J. C., BACCARI JÚNIOR, F.; TARGA, L. A. Respostas fisiológicas e produtivas de vacas holandesas com acesso à sombra constante ou limitada. Revista Brasileira de Zootecnia, Viçosa v. 27, n. 3, p. 595-602, 1998.

FRAZZI, E.; CALAMARI, L.; CALEGARI, F. Different systems of air conditioning for dairy cows housing in Mediterranean climate. In: International Congress on Agricultural Engineering, 130. Marrocos, 1998, p.23-29.

GAYÃO, A.L.B.A. 1992. Efeito do estresse térmico sobre a taxa metabólica e o desempenho produtivo de cabritas Saanen em crescimento. Dissertação (Mestrado em Zootecnia). Faculdade de Medicina Veterinária e Zootecnia. Universidade Estadual Paulista. Botucatu. 68 $\mathrm{pp}$

GEBREMEDHIN, K. G.; WU, B. Simulation of sensible and latent heat losses from wet-skin surface and fur layer. Journal of Thermal Biology, Oxford, v. 27, p. 291-297, 2002.

ESMAY, M.L. Principles of animal environment. Westport : Avi, 1982. 325p.

HALL, W.B., MCKEON, G.M., CARTER, J.O., DAY, K.A., HOWDEN, S.M., SCANLAN, J.C., JOHNSTON, P.W. AND BURROWS, W.H. Climate change and Queensland's grazing lands: II. An assessment of the impact on animal production from native pastures. Rangeland Journal 20, 174-202, 1998.

HAHN, G.L.; PARKHURRST, A.M.; GAUGHAN, J.B. Cattle respiration rate as a function of ambient temperature.Transactions of American Society of Agricultural Engineering, v.40, p.97-121, 1997.

IGONO, M.O.; STEEVENS, B.J.; SHANKLIN, M.D. et al. Spray cooling effects on milk production, milk and rectal temperature of cows during a moderate temperature summer season. Journal of Dairy Science, Wisconsin, v.68, p.979-985. 1985.

JOHNSON, H.D. Bioclimates and Livestock. In: World Animal Science, Bioclimatology and the adaptation of livestock pp. 3-16. Edited by Johnson H.O., Elsevier, Amsterdam, 1987.

KELLY, W.R. Veterinery clinical diagnosis. London: Bailliére Tindal and Cassel,1967.p. 247

MAIA, A.S.C.; SILVA, R.G.; LOUREIRO, C.M.B. Sensible and Latent Heat Loss from the Body Surface of Holstein Cows in a Tropical Environment. International Journal of Biometeorology, v.50, n.1, p.17-22, 2005.

MARTELLO, L. S.; SILVA, S. L.; TITTO, E. A. L. Respostas fisiológicas e produtivas de vacas holandesas em lactação submetidas a diferentes ambientes. Revista Brasileira de Zootecnia, v.33, n.1, p.181-191, 2004. 
McDOWELL, R.E.; HOOVEN, N.M.; CAMOENS, J.K. Effect of climate on performance of Holstein in first lactation. Journal of Dairy Science, v.59, n.5, p.965-973, 1976.

MOTA, L.S.L.S. Adaptação e interação genótipo-ambiente em vacas leiteiras. Ribeirão Preto: Universidade de São Paulo, 1997. Tese (Doutorado em Ciências) - Universidade de São Paulo, 1997.

NÄÄS, I. DE A. Princípios de conforto térmico na produção animal. São Paulo: Icone Ed.,1989. 183p.

NÄÄS, I.A.; ARCARO JR., I. Influência de ventilação e aspersão em sistemas de sombreamento artificial para vacas em lactação em condições de calor. Revista Brasileira de Engenharia Agrícola e Ambiental, v.5, n. 1, p. 139-142, 2001.

PERISSINOTTO, M.; MOURA, D.J.; MATARAZZO, S.V. et al. Behavior of dairy cows housed in environmentally controlled freestall. Agricultural Engineering International: the CIGR Ejournal, v.3, p.5-16, 2003.

RODRIGUEZ, T. Patologia general y exploración clínica de los animales domesticos. 3 ed.. Barcelona: Labor, 1948. p..325.

ROMAN-PONCE, H.; THATCHER, W.W.; BUfFINGTON, D.E. et al. Physiological and production responses of dairy cattle to a shade structure in a subtropical environment. Journal of Dairy Science, v.60, p.424-30, 1977.

ROMA JR., L.C.; SILVA, I.J.O.; PINHEIRO, M.G.; PIEDADE, S.M.S. Avaliação física do sistema de resfriamento adiabático evaporativo (SRAE) em instalações do tipo freestall para bovinos de leite. In: CONGRESSO BRASILEIRO DE ENGENHARIA AGRÍCOLA, 23. Foz do Iguaçu. Anais... Foz do Iguaçu, 2001.

SILVA I.J.O Climatização das instalações para bovino leiteiro. In: SIMPÓSIO BRASILEIRO DE AMBIÊNCIA NA PRODUÇÃO DE LEITE, 1., Piracicaba, 1998. Anais... Piracicaba: FEALQ, p. 114-145, 1998.

SILVA, R. G. Estimativa do balanço térmico por radiação em vacas Holandesas expostas ao sol e á sombra em ambiente tropical. Revista Brasileira de Zootecnia, Viçosa, v. 28, n. 6, p. 1403-1411, 1999.

SILVA R. G. Introdução à bioclimatologia animal. São Paulo: Nobel, 2000. 286p.

SILVA R. G.; GUILHERMINO M. M.; MORAIS D. A. E. F. Thermal radiation absorbed by dairy cows in pasture. Int J Biometeorol 54:5-11, 2010.

SCHMIDT-NIELSEN, K. Fisiologia animal: adaptação e ambiente, 5.th ed. Cambridge: Cambridge Univ. Press, 2002, 611p.

SILVA, R. B.; SOUZA JR, J. B. F.; DOMINGOS, H. G. T.; MAIA, A. S. C. Variação circadiana da temperatura retal, da superfície do pelame e da freqüência respiratória em vacas holandesas manejadas em ambiente tropical numa região semi-árida. In: REUNIÃO ANUAL DA SOCIEDADE BRASILEIRA DE ZOOTECNIA, 45., Lavras,MG. Anais..., Lavras,MG, 2008.

SOUZA JR, J.B.F.; SILVA, R. B.; DOMINGOS, H. G. T.; MAIA, A. S. C. Temperatura da superfície corporal e fluxo de calor convectivo em vacas Holandesas expostas à radiação solar direta no Semi-Árido. In: V CONGRESSO NORDESTINO DE PRODUÇÃO ANIMAL, ARACAJÚ/SE. ANAIS..., 2008. 
TITTO, E.A.L. Clima: influência na produção de leite. Piracicaba, SP, 1998. In: SIMPÓSIO BRASILEIRO DE AMBIÊNCIA NA PRODUÇÃO DE LEITE, 1., 1998, Piracicaba, SP. Anais... Piracicaba: FEALQ, 1998. p. $10-23$. 